\title{
Magnetic shadowing of high energy ions at Mars and how this effect can be simulated using a hybrid model
}

\author{
S. McKenna-Lawlor ${ }^{1}$, E. Kallio ${ }^{2}$, R. Jarvinen ${ }^{2}$, and V. V. Afonin ${ }^{3}$ \\ ${ }^{1}$ Space Technology Ireland, Maynooth, Co. Kildare, Ireland \\ ${ }^{2}$ Finnish Meteorological Institute, Helsinki, Finland \\ ${ }^{3}$ Space Research Institute, Moscow, Russia
}

(Received February 4, 2011; Revised June 5, 2011; Accepted June 20, 2011; Online published March 8, 2012)

\begin{abstract}
Energetic particle data recorded by the SLED instrument aboard Phobos-2 while in circular orbit about Mars (6-26 March, 1989) showed the presence of magnetic shadowing. A 3-D, self consistent, hybrid model (HYBMars) supplemented by test particle simulations was developed to study the response of the Martian plasma environment to solar disturbances and to reproduce, in particular, the magnetic shadowing effect. The pertaining magnetic and electric fields as well as the properties of high energy ions present at Mars under conditions of extreme solar disturbances, can be derived from HYB-Mars. This model predicted a plasma phenomenon at the planet, named here 'solar wind-flow shadowing', which was earlier identified in the measurements of the ASPERA (plasma) experiment aboard Phobos-2. HYB also predicted magnetic shadowing which is qualitatively similar to that recorded by SLED. The simulations suggest that the configuration of a magnetic shadow depends on the pertaining solar wind density and velocity, and on the magnitude and direction of the interplanetary magnetic field. It is currently planned to input to the HYB model plasma and magnetic field data measured contemporaneously with the particle measurements aboard Phobos-2 so as to more realistically match the simulated results with the in situ observations.
\end{abstract}

Key words: Mars, hybrid model, magnetic shadow.

\section{Introduction}

Studies of spacecraft measurements made over several decades have made it possible to establish criteria that enable records of transient magnetized plasma flows in the interplanetary medium to be reliably identified as the consequences of Coronal Mass Ejections, CMEs. To distinguish CMEs in the solar wind from their solar counterparts, the former events are termed Interplanetary Coronal Mass Ejections, ICMEs (e.g. Gosling, 1990; Cane and Richardson, 2003). It was demonstrated by Reames (1999) that the particle profile associatively recorded in space depends on where an observer is located relative to the moving shock source. Due to the fact that the relative positions of the Sun, Earth and Mars are constantly changing, while also CMEs are often strongly directional, it can be the case that some significant energetic particle events recorded at Earth in association with ICMEs either produce very weak particle signatures at Mars or do not arrive there at all. In other geometries, energetic particles that impact Mars directly due to conditions of good magnetic connectivity may not impact Earth.

Given that the prevailing geometry is appropriate for ICME arrival, changing ambient conditions can be expected to characterize the period associated with the incidence of

Copyright (c) The Society of Geomagnetism and Earth, Planetary and Space Sciences (SGEPSS); The Seismological Society of Japan; The Volcanological Society of Japan; The Geodetic Society of Japan; The Japanese Society for Planetary Sciences; TERRAPUB.

doi:10.5047/eps.2011.06.039 such an event at Mars. A possible scenario could, for instance, feature the initial arrival of anisotropic (beamed) particles, followed by increased dynamic pressure and by the enhanced magnetic field associated with ICME arrival. Such complex occurrences which, depending on the level of parent solar activity, may involve the arrival of a sequence of similarly directed ICMEs rather than a single event, can potentially be associated with long term consequences at Mars in terms of magnetic draping and magnetic shadowing (see below). This indicates the importance of making detailed simulations of such phenomena in order to gain insight into the response of Mars to changing solar circumstances.

\subsection{The present study}

The motivation for the present study was to model a data set composed of energetic protons with energies in the range $30 \mathrm{keV}$ to a few tens of $\mathrm{MeV}$ recorded at Mars by the SLED (Solar Low Energy Detector) particle detector aboard the Phobos-2 spacecraft under disturbed solar conditions in March, 1989 (McKenna-Lawlor et al., 1992, 2005). To achieve this aim a 3-D, self-consistent, hybrid model (HYB), supplemented by test particle simulations, was developed to investigate the macroscopic properties of solar related high energy ion populations near Mars and to study the motion of individual high energy ions at the planet. In addition to modelling the nominal solar wind $\sim 1 \mathrm{keV} \mathrm{H}^{+}$ ion population, HYB simulations of four high energy solar wind $\mathrm{H}^{+}$populations in the energy range $50 \mathrm{keV}-3.2$ $\mathrm{MeV}$ were performed and comparisons made with SLED 
data recorded on 13 March, 1989.

Section 2 describes energetic particle data sets recorded thus far at Mars. In Section 3 an account of the SLED particle detector aboard Phobos- 2 is provided. Also, an overview of the disturbed energetic particle environment at Mars associated with major flaring on the Sun in March, 1989 is presented, together with an account of the associated detection in SLED measurements of magnetic shadowing at Mars. Section 4 provides an overview of the HYBmodel and accompanying test particle simulations developed for the present study. Section 5 introduces predictions of the Martian high energy ion environment generated by HYB and shows how they compare with SLED in situ measurements made on 13 March, 1989. Section 6 contains a discussion and Section 7 conclusions.

\section{Energetic Particle Data Sets Recorded at Mars}

Pioneering measurements of the high energy particle environment at Mars were made by the SLED instrument aboard Phobos-2. SLED measured ions with energies in the range $\sim 30 \mathrm{keV}$ to a few tens of $\mathrm{MeV}$. That part of the data set considered here was recorded while the spacecraft was in circular orbit about Mars at a height above the planet of $\sim 6300 \mathrm{~km}$ on 13 March, 1989 .

A second set of high energy particle observations was obtained at Mars by the MARIE (Mars Radiation Environment Experiment) aboard NASA's Mars Odyssey spacecraft (Zeitlin et al., 2004). MARIE measured ions with energies in the range $(\sim 20-200 \mathrm{MeV})$ while in circular orbit about the planet at a height of $\sim 400 \mathrm{~km}$ (Section 6.2).

The next opportunity to measure high energy ions at Mars will be realized (a) by the Radiation Assessment Detector (RAD) to be flown on the Mars Science Laboratory, MSL (scheduled for launch in 2011) which is designed to measure solar energetic particles aboard a rover at the Martian surface and (b) by the Solar Energetic Particle (SEP) experiment which is scheduled for launch on the Maven Mission in 2013.

\section{The Phobos-2 Mission and the Onboard SLED Instrument}

Phobos-2 was launched to Mars from the Baikonur Cosmodrome on 12 July, 1988 and reached Mars on 29 January, 1989. The spacecraft was transferred on 1 February into a series of four elliptical planetary orbits (pericenter, 867 $\mathrm{km}$ ). It was next transferred to an elliptical orbit with high pericenter, then to a circular equatorial orbit (altitude $\sim 6,330 \mathrm{~km}$ ) in which it circled the planet 114 times. Ground connection with the spacecraft was lost at the end of March (Sagdeev and Zakharov, 1989). The SLED data studied here were acquired while the spacecraft was executing circular equatorial orbits about Mars on 13 March, 1989.

In the course of the 114 circular planetary orbits performed during the encounter of Phobos-2 with Mars, the Bow Shock (BS), Magnetic Pileup Boundary (MPB) and the Magnetotail were traversed multiple times. It is recalled that the MPB is defined to comprise a sharp, thin and welldefined plasma boundary located between the bow shock and the inner ionospheric boundary at comets, Mars and
Venus (Bertucci et al., 2005). This boundary separates the magnetosheath (a region of low magnetic fields characterized by conspicuous wave activity) from the magnetic pile up region - which is dominated by strong, highly organized magnetic fields resulting from the pileup and draping of the interplanetary magnetic field. A variety of names have been employed in the literature to designate this boundary (see Vignes et al., 2000) who used comparative data to show that the magnetic pileup boundary, planetopause, magnetopause, plasma composition boundary and protonopause are all names for the same feature. A detailed account of the issues involved is contained in Nagy et al. (2004). See also an account of the further name Induced Magnetospheric Boundary (IMB) used by the Mars Express experimenters to describe the boundary between the Martian magnetosheath (where the solar wind plasma is diverted around the planet) and the ionosphere/tail/inner magnetosphere (Lundin et al., 2004). It was pointed out in this regard by Winningham et al. (2006) that the IMB is located at, or very near, the magnetic pile-up boundary, the inherent difference being that the MPB is defined using its magnetic field signature whereas the IMB is defined using its particle signature.

\subsection{The SLED instrument}

The charged-particle detector SLED aboard Phobos-2 was designed to simultaneously measure fluxes of ions and electrons in the $\sim 30 \mathrm{keV}$ to a few $\mathrm{MeV}$ range. The instrument employed two semiconductor detectors in each of two telescopes (named Te-1 and Te-2), both of which viewed in the same direction $\left(55^{\circ}\right.$ westward of the Sun-Earth linethat is approximately in the nominal direction of the interplanetary magnetic field at Mars).

The front detector of Te-1 was covered with a $15 \mu \mathrm{g} / \mathrm{cm}^{2}$ Al foil. Te-2 used in addition an Al foil $\left(500 \mu \mathrm{g} / \mathrm{cm}^{2}\right)$ which absorbed protons with energies $<350 \mathrm{keV}$ while allowing the detection of 35-350 keV electrons. The count rate differences between Te- 1 and Te- 2 allow proton and electron fluxes to be differentiated following the method of Anderson et al. (1978). Table 1 presents the energy channels of SLED.

The geometric factor of each telescope was $0.21 \mathrm{~cm}^{2}$ ster; the Field of View (FOV) axis, with a $40^{\circ}$ apex angle,

Table 1. Energy channels of SLED.

\begin{tabular}{lll}
\hline \multicolumn{2}{c}{ Te 1 (without foil) } \\
\hline Ch. 1 & $30-50 \mathrm{keV}$ & electrons + ions \\
Ch. 2 & $50-200 \mathrm{keV}$ & electrons + ions \\
Ch. 3 & $200-600 \mathrm{keV}$ & electrons + ions \\
Ch. 4 & $0.6-3.2 \mathrm{MeV}$ & ions \\
Ch. 5 & $3.2-4.5 \mathrm{MeV}$ & ions \\
Ch. 6 & $>30 \mathrm{MeV}$ & background rate \\
\hline & \multicolumn{2}{c}{ Te 2 (with foil) } \\
\hline Ch. 1 & $30-50 \mathrm{keV}$ & electrons $+350-400 \mathrm{keV}$ ions \\
Ch. 2 & $50-200 \mathrm{keV}$ & electrons $+400-500 \mathrm{keV}$ ions \\
Ch. 3 & $200-600 \mathrm{keV}$ & electrons $+0.5-1 \mathrm{MeV}$ ions \\
Ch. 4 & $0.6-3.2 \mathrm{MeV}$ & ions \\
Ch. 5 & $3.2-4.5 \mathrm{MeV}$ & ions \\
Ch. 6 & $>30 \mathrm{MeV}$ & background rate
\end{tabular}




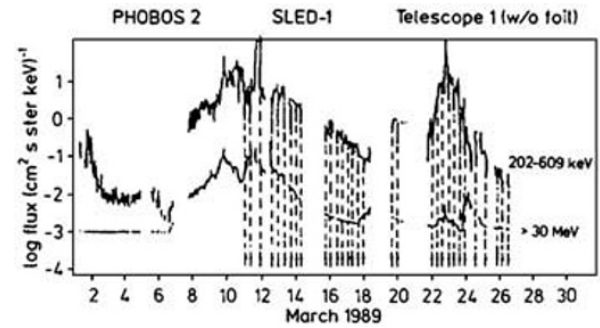

Fig. 1. Enhanced particle fluxes recorded by SLED in Channels 3 and 6 during March, 1989 in association with major ongoing flaring. Significant depressions in flux due to magnetic shadowing are indicated by dashed vertical lines. Following McKenna-Lawlor et al. (1991).

was in the ecliptic plane at $55^{\circ}$ to the west of the sunward direction and the time resolution was $230 \mathrm{~s}$. For details of the instrument see McKenna-Lawlor et al. (1990).

\subsection{The particle environment at Mars (March, 1989)}

NOAA Active Region 5395 rotated onto the solar disk on 6 March and, thereafter, quickly produced a $15 \mathrm{X} / 3 \mathrm{~B}$ flare at 13.54 UT. Thereafter, significant particle associated flaring continued until $>26$ March when contact with the spacecraft was lost. Phobos-2, which had already entered a circular $\left(8^{\mathrm{h}}\right)$ equatorial orbit about Mars when this active episode on the Sun began, flew alternately in the solar wind and inside the Martian bow shock-where it executed repeated traversals of the Martian magnetosphere. Against the general background of energetic particle and shock signatures associated with ongoing flaring, the particle records obtained by SLED displayed several characteristic signatures associated with crossing the magnetosphere. Among these was a sequence of narrow drops due to 'magnetic shadowing' of the field of view of the instrument. Another was a series of sharp increases due to ambient particle acceleration as the spacecraft traversed the Martian bow shock, inbound and outbound. In Fig. 1, depressions due to magnetic shadowing of the fluxes recorded in Channels 3 and 6 of Te-1 (SLED) during March 1989 are indicated by dashed lines.

A more detailed example of a representative depression in counts recorded by SLED in Channel 4 of Te-1 (0.63.2 MeV) on 13 March, 1989 (00:00-08:00 UT) during a circular planetary orbit is displayed in Fig. 2 (represented in Mars-Sun Orbit/MSO coordinates: $+x$ sunward direction, $+z$ to the north ecliptic pole, $+y$ completes the righthanded system). The look direction of SLED is indicated at the top/right. The Field of View of SLED was relatively well aligning with the IMF which was quite Parker spiral like on 13 March $\left(A_{z}=130^{\circ}-160^{\circ}\right.$; nominal $\left.A_{z}=120^{\circ}\right)$ figure not shown. Mars is represented at the center of each plot. The relative number of counts recorded at successive orbital locations is indicated by the magnitude of vertical lines that represent the individual readings. Gaps correspond to operational switch off times occasioned by telemetry constraints or loss of data. The depression is due to particle shadowing (McKenna-Lawlor et al., 1991).

\section{Overview of the HYB-Mars Hybrid Model}

Numerical simulations were performed by the global, self-consistent, hybrid model HYB-Mars which was devel- oped at the Finnish Meteorological Institute. In a hybrid model ions are modelled as particles whereas electrons form a massless, charge neutralized, fluid. A detailed description of the HYB-Mars model is published elsewhere (Kallio et al., 2010) and, in the present text, only basic features relevant to the present study are described.

The coordinate system used in the HYB-Mars model is MSO: the $x$-axis points from the center of Mars to the Sun; the $z$-axis is perpendicular to the orbital plane of Mars pointing toward the north ecliptic pole, and the $y$-axis completes the right-handed coordinate system. When the response of the Martian plasma environment to upstream solar wind conditions was studied (see below), the size of the simulation box used was $-14,400 \mathrm{~km}<x, y, z<$ $14,400 \mathrm{~km}$ (that is approximately $-4 R_{\mathrm{M}}<x, y, z<4 R_{\mathrm{M}}$ ), where $R_{\mathrm{M}}$ is the radius of Mars $(3393 \mathrm{~km})$. The hierarchically refined cubic grid adopted had three grid sizes, depending on the distance from the center of Mars $(r)$ involved: $0.2 R_{\mathrm{M}}$ at $r>3 R_{\mathrm{M}}, 0.1 R_{\mathrm{M}}$ at $2 R_{\mathrm{M}}<r<3 R_{\mathrm{M}}$, and $0.05 R_{\mathrm{M}}$ at $r<2 R_{\mathrm{M}}$. The time step used in the simulations (dt) was $0.02 \mathrm{~s}$.

In the 'solar wind response runs', four different upstream parameters were utilized. The nominal run ${ }^{1}$ was compared (Table 2) with two runs made when the dynamic pressure of the solar wind was increased by a factor of four, either by doubling the speed of the solar wind (the high velocity solar wind $2 \times U_{\mathrm{sw}}$ run) or by increasing the solar wind density by a factor of four (the high density solar wind $4 \times n_{\text {sw }}$ run). Finally, the role of the magnitude of the interplanetary magnetic field (IMF) was investigated in the 'high density and high IMF' run by increasing the magnetic field used in the high density run by a factor of four. These various runs were designed to provide insight into how the disturbed solar wind affects, either through an increase in its density, velocity or IMF, the Martian plasma environment, in particular its ambient high energy protons.

Next, the motion of the high energy proton populations was studied with respect to each of the four upstream cases selected. The energies of the four high energy solar wind $\mathrm{H}^{+}$beams were individually taken to be $50 \mathrm{keV}, 200 \mathrm{keV}$, $600 \mathrm{keV}$ and $3.2 \mathrm{MeV}$. These energies were chosen because they correspond to the highest particle energies recorded in Te-1, Channels 1-4, of the SLED instrument (Table 1), thereby enabling an investigation to be made as to how the magnetic shadowing detected by SLED depended on ion energy.

In the simulation process, the high energy $\mathrm{H}^{+}$ions and the main $\mathrm{H}^{+}$population were each injected into the simulation box at $x=10,800 \mathrm{~km}\left(\sim 3 R_{\mathrm{M}}\right)$. The four high energy beams then simulated the high energy tail in the solar wind velocity distribution function. The density of these high energy ions was set at a sufficiently low value that the selfconsistent solution was not affected. In order to specify the main solar wind $\mathrm{H}^{+}$ion population (which in velocity space is located near the bulk velocity), it is referred to hencefor-

\footnotetext{
${ }^{1}$ The upstream solar wind parameters selected for the nominal runs will again be used as input parameters during the next international Solar Wind Interaction with Mars, SWIM, modeling challenge when several hybrid models and MHD models will be compared. See Brain et al. (2010) for an account of the first SWIM modeling comparisons.
} 
Table 2. The density $\left(n_{\mathrm{sw}}\right)$ and bulk velocity $\left(U_{\mathrm{sw}}\right)$ of the solar wind protons and the strength of the interplanetary magnetic field IMF $\left(\boldsymbol{B}_{\mathrm{sw}}\right)$ used in the five upstream parameter runs analyzed.

\begin{tabular}{lccc}
\hline Name of the run & $\boldsymbol{n}_{\mathrm{sw}}\left[\mathrm{cm}^{-3}\right]$ & $\boldsymbol{U}_{\mathrm{sw}}\left[\mathrm{km} \mathrm{s}^{-1}\right]$ & $\boldsymbol{B}_{\mathrm{sw}}[\mathrm{nT}]$ \\
\hline Nominal & 2.7 & {$[-485,0,0]$} & {$[-1.634,2.516,0]$} \\
High velocity $\left(2 \times U_{\mathrm{sw}}\right)$ & 2.7 & {$[-\mathbf{9 7 0 , 0 , 0}]$} & {$[-1.634,2.516,0]$} \\
High density $\left(4 \times n_{\mathrm{sw}}\right)$ & $\mathbf{1 0 . 8}$ & {$[-485,0,0]$} & {$[-1.634,2.516,0]$} \\
High density and high IMF $\left(4 \times n_{\mathrm{sw}}\right)$ and $(4 \times \mathrm{IMF})$ & $\mathbf{1 0 . 8}$ & {$[-485,0,0]$} & {$[-\mathbf{6 . 5 3 6 , 1 0 . 0 6 4 , 0}]$} \\
\hline
\end{tabular}

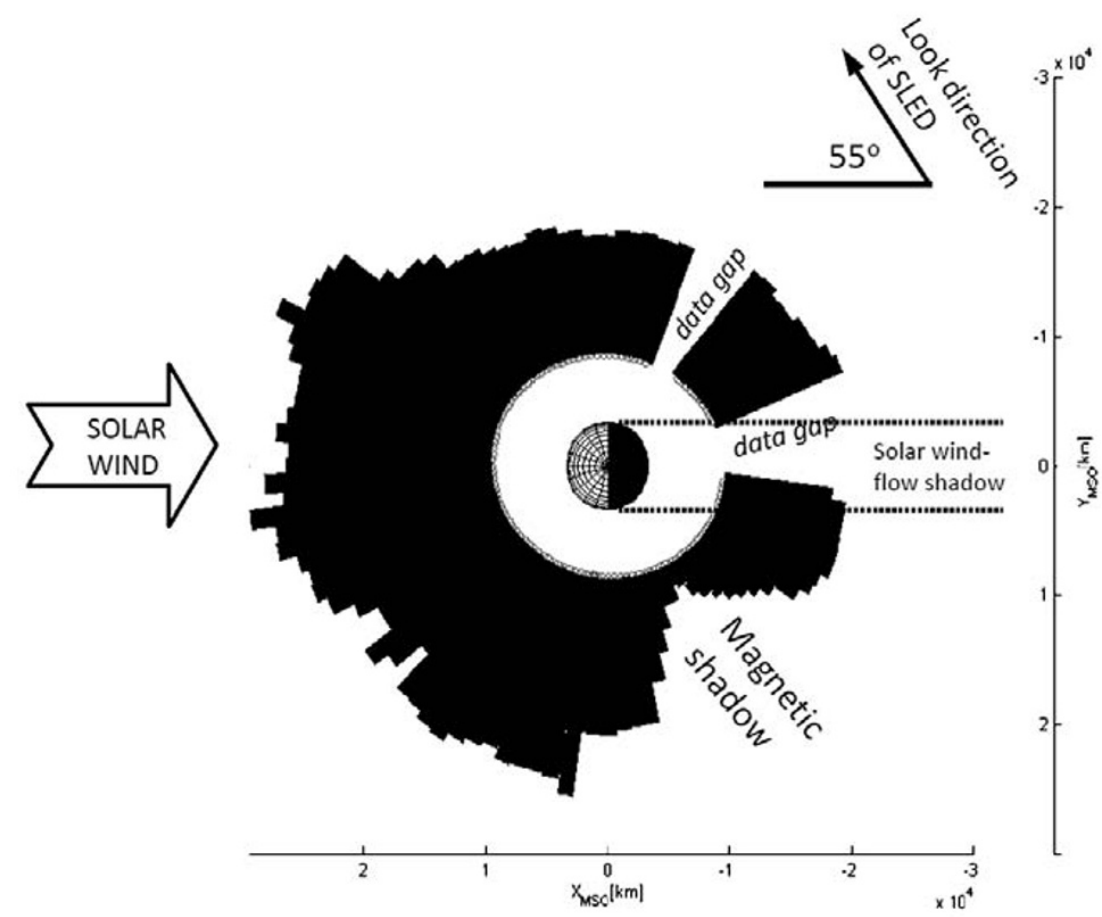

Fig. 2. An example of counts recorded by SLED in Channel 4 of Te-1 (0.6-3.2 MeV) on 13 March, 1989 (00:00-08:00 UT) while traversing a circular planetary orbit. The coordinate system is MSO: $+x$ sunward direction, $+z$ to the north ecliptic pole, $+y$ completes the right handed system. The look direction of SLED is indicated at the top/right. Mars is represented at the centre of the plot. The relative number of counts recorded along particular orbits is indicated by the magnitude of lines that represent the individual readings made at successive locations. These lines are drawn from points along the orbital path in a direction perpendicular to that path. Gaps correspond to operational switch off times or loss of data and the depression is due to particle shadowing. The two dotted lines behind Mars indicate the location of the solar wind flow shadow caused by the main $\mathrm{H}^{+}$population in the solar wind near $\sim 1 \mathrm{keV}$.

ward here as $\mathrm{H}_{\mathrm{sw}}+$ ions. Similarly, the four high energy $\mathrm{H}^{+}$populations are referred to as: $\mathrm{H}_{\mathrm{h} 1}+(50 \mathrm{keV}), \mathrm{H}_{\mathrm{h} 2}{ }^{+}$ (200 keV), $\mathrm{H}_{\mathrm{h} 3}{ }^{+}(600 \mathrm{keV})$ and $\mathrm{H}_{\mathrm{h} 4}{ }^{+}(3.2 \mathrm{MeV})$.

In the high energy $\mathrm{H}^{+}$ion runs the sizes adopted for the simulation box were: $-10,800 \mathrm{~km}<x<10,800 \mathrm{~km}$; $-43,200 \mathrm{~km}<y<10,800 \mathrm{~km} ;-28,800 \mathrm{~km}<z<$ 21,600 km (i.e., approximately: $-3 R_{\mathrm{M}}<x<3 R_{\mathrm{M}}$; $\left.-12 R_{\mathrm{M}}<y<3 R_{\mathrm{M}} ;-8 R_{\mathrm{M}}<z<6 R_{\mathrm{M}}\right)$. A box size elongated in the $y$-direction was utilized in order to ensure that the high energy ions, which in this study are, for simplicity, launched exactly along the IMF (see discussions about this approximation in Section 6.3), filled the region of interest at $-3 R_{\mathrm{M}}<x, y, z<-3 R_{\mathrm{M}}$. An elongated size in the $z$ direction was adopted in order to ensure that the high energy protons did not hit the $z$-faces of the simulation box during their gyro-motions. In this box only one grid size $\left(0.2 R_{\mathrm{M}}\right)$ was used in order to maintain a feasible simulation time, despite the large size of the space. The time step of the simulation $(d t)$ was initially taken to be $0.04 \mathrm{~s}$. However, after the motion of the high energy ions was initially studied, this time step was decreased to $0.002 \mathrm{~s}$ in order to ensure that the high energy ions moved through a lesser distance than the smallest grid size used $(\sim 180 \mathrm{~km})$ during the time step selected.

\section{Predictions of the HYB Model}

In this section the way in which Mars affects ambient solar wind $\mathrm{H}^{+}$ions under different solar wind conditions is investigated using the HYB model.

\subsection{Response of the Martian plasma environment to upstream solar wind conditions}

The hybrid model provides a possibility to study the response of the Martian plasma environment to various disturbed upstream parameters. The four color plots making up Fig. 3 show, for each case analysed, the density of protons in the noon-midnight meridian plane. In every figure a bow shock, visible as an increase in density above the planet, can be identified. A notable feature in Fig. 3 is the formation of a low density $\mathrm{H}_{\mathrm{sw}}+$ ion region behind Mars around the Mars-Sun axis. This predicted density decrease 

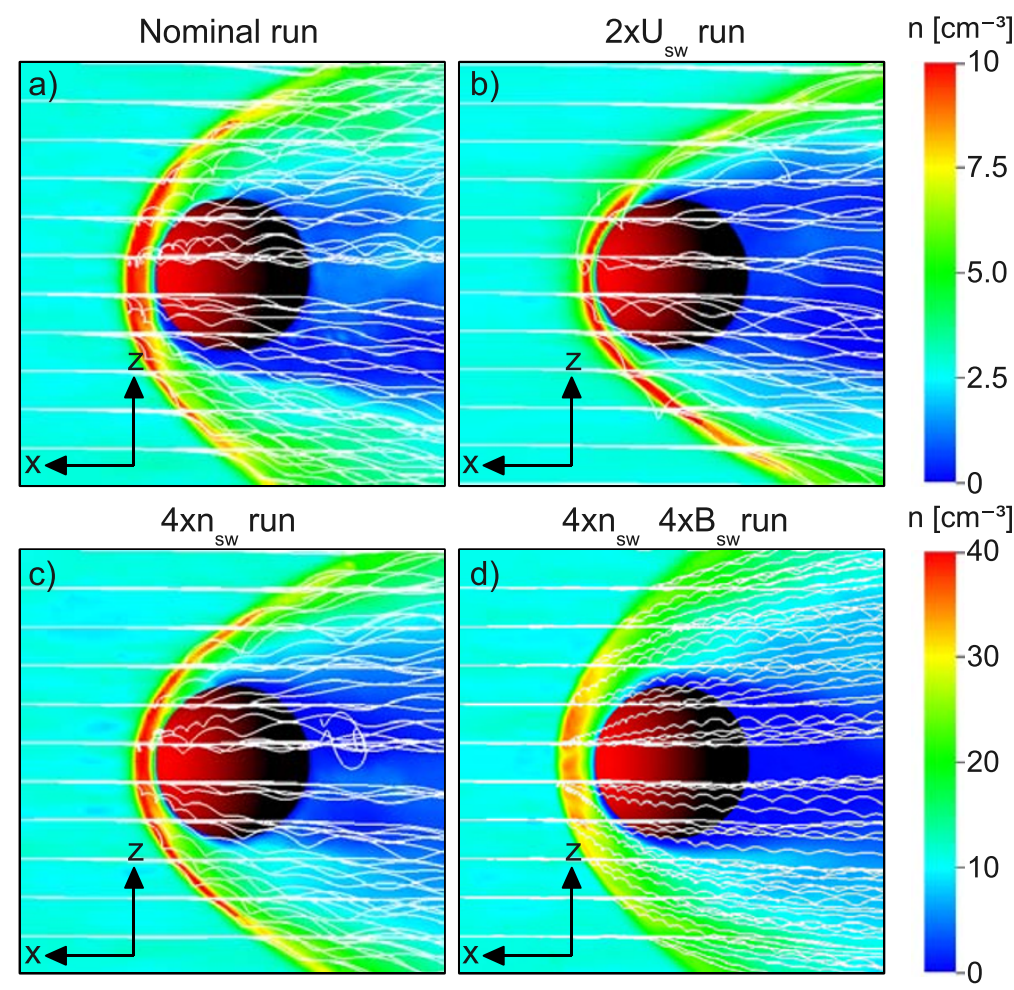

Fig. 3. The motion of solar wind $\mathrm{H}_{\mathrm{sw}}{ }^{+}$ions which have the solar wind bulk velocity in four upstream cases: (a) the nominal run where $U_{\mathrm{sw}}=485 \mathrm{~km} \mathrm{~s}^{-1}, n_{\mathrm{sw}}=2.7 \mathrm{~cm}^{-3}, \mathrm{IMF}=[-1.634,2.516,0] \mathrm{nT}$, (b) the high velocity $2 \times U_{\mathrm{sw}}$ run where $U_{\mathrm{sw}}=970 \mathrm{~km} \mathrm{~s}^{-1}, n_{\mathrm{sw}}=2.7 \mathrm{~cm}^{-3}$, $\mathrm{IMF}=[-1.634,2.516,0] \mathrm{nT}$, (c) the high density $4 \times n_{\mathrm{sw}}$ run where $U_{\mathrm{sw}}=485 \mathrm{~km} \mathrm{~s}^{-1}, n_{\mathrm{sw}}=10.8 \mathrm{~cm}^{-3}, \mathrm{IMF}=[-1.634,2.516,0] \mathrm{nT}$ and (d) the high density and high IMF $4 \times n_{\mathrm{sw}} 2 \times \mathrm{IMF}$ run where $U_{\mathrm{sw}}=485 \mathrm{~km} \mathrm{~s}^{-1}, n_{\mathrm{sw}}=10.8 \mathrm{~cm}^{-3}, \mathrm{IMF}=[-6.536,10064,0] \mathrm{nT}$. The initial temperature of the test particles was zero and they were generated near the $x=10,000 \mathrm{~km}$ plane on the 15 ( $y$-direction) $\times 15$ ( $z$-direction) grid. The color in the $y=0$ noon-midnight meridian planes shows the total density of protons. The white lines show test particle orbits. The horizontal and the vertical lengths are each from $-10,000 \mathrm{~km}$ to $10,000 \mathrm{~km}$. Note the presence of the low density $\mathrm{H}_{\mathrm{sw}}{ }^{+}$"solar wind-flow shadow" behind the planet (dark blue areas).
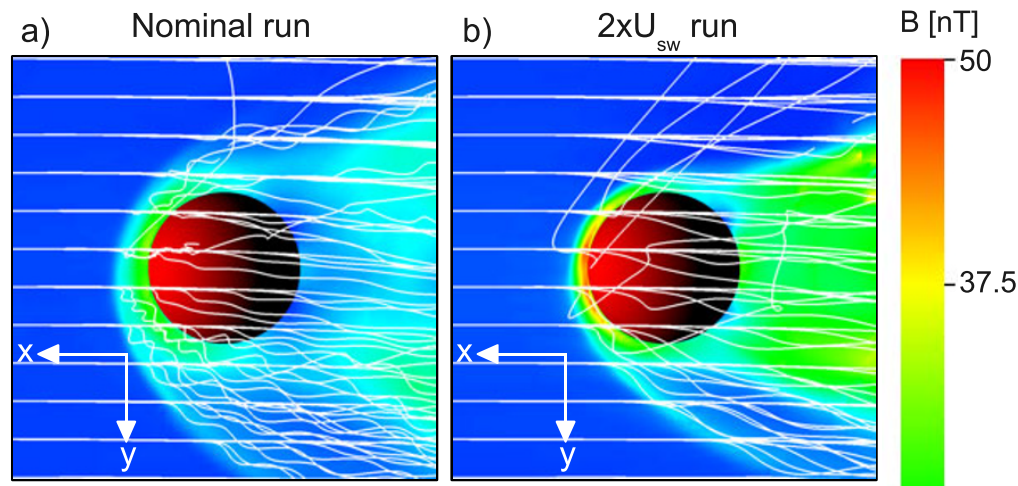

c)

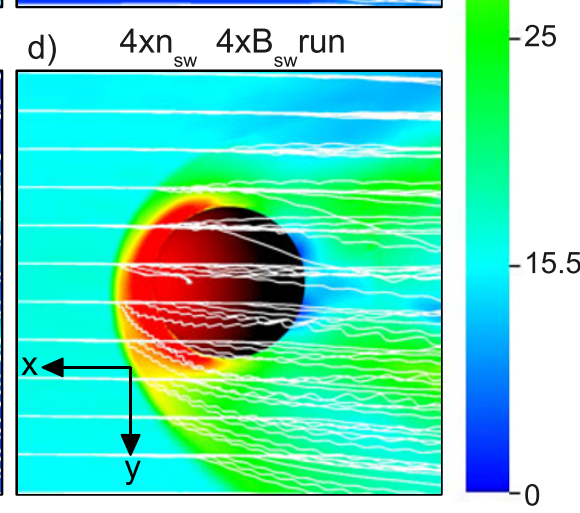

Fig. 4. Motion of the solar wind $\mathrm{H}_{\mathrm{sw}}{ }^{+}$test particles in the four upstream cases already considered in Fig. 3: (a) the nominal run, (b) the high velocity $2 \times U_{\text {sw }}$ run, (c) the high density $4 \times n_{\text {sw }}$ run and (d) the high density and high IMF $4 \times n_{\text {sw }} 2 \times$ IMF run. The trajectories are the same as in Fig. 3 but they are now viewed along the $z$-axis above the northern hemisphere. The color planes in the $z=0$ equator plane indicate the magnitude of the magnetic field. The horizontal and the vertical lengths are each from $-10,000 \mathrm{~km}$ to $10,000 \mathrm{~km}$. 
behind Mars is called in the present paper "a solar windflow shadow" because it is located on the opposite side of the planet from that at which the solar wind flow impacts Mars. It is pointed out in addition that the simulation places this shadow at that distance from the planet where the ASPERA instrument aboard Phobos-2 measured a substantial decrease in the flux of $\mathrm{H}^{+}$ions behind Mars (see for instance Kallio et al., 1994). Thus, this prediction of the model is validated by the ASPERA/Phobos- 2 observations.

Test particles are utilized in Fig. 3 to illustrate kinetic effects. In all four cases the gyro-radius of each $\mathrm{H}_{\mathrm{sw}}{ }^{+}$ion with velocity $U_{\mathrm{sw}}$ is so large that this ion gyro-motion can be discerned in the plots by eye although, in every instance, it is significantly smaller than the radius of Mars. Under such circumstances the flow of the main solar wind $\mathrm{H}^{+}$ population around Mars is quite "fluid-like".

In Fig. 4 the trajectories of the same test particles are viewed along the $+z$ axis. In this viewing direction, protons are seen to move in a, relatively coherent, fluid-like manner despite the fact that some $\mathrm{H}^{+}$ions are reflected toward the dawn hemisphere $(y<0)$ at those locations where the IMF is parallel to the normal to the bow shock. The color plots in Fig. 4 show the total magnetic field, which ultimately turned out (see below) to be the most important, single, physical variable involved when the motion of high energy ions was studied (because of the $e \boldsymbol{v}_{\mathrm{i}} \times \boldsymbol{B}$ term, i.e. the Lorentz force).

In all cases, the highest magnetic field was present near the planet on the dayside where the IMF "piled up" against Mars. On the night-side the draped IMF formed two magnetic tail lobes, which appear in Fig. 4 as an enhanced magnitude in the magnetic field. A comparison between the high velocity (Fig. 4(b)) and the nominal runs (Fig. 4(a)) shows that the increased solar wind velocity "compressed" the bow shock and the magnetosphere on both the dayside and the nightside. A similar compression is present in the density data (compare Figs. 3(a) and 3(b)). Simultaneously, the magnitude of the magnetic field increased.

In the high density run plot (Fig. 4(c)), the dynamic pressure of the solar wind was, as in the high velocity case (Fig. 4(b)), four times higher than nominal. However, the increased solar wind density affects very little the total magnetic field (see Fig. 4(c)). In this regard the highest magnetic field can be seen in Fig. 4(d) where the solar wind density is the same as that in the high density run (Fig. 4(c)) but where the magnitude of the IMF was increased by a factor of four.

\subsection{High energy protons near Mars}

The motions of $50 \mathrm{keV}, 200 \mathrm{keV}, 600 \mathrm{keV}$ and $3.2 \mathrm{MeV}$ $\mathrm{H}^{+}$ions are illustrated in Fig. 5. Unlike what was seen in the case of the $\mathrm{H}_{\mathrm{sw}}{ }^{+}$ions, the ion gyro-radius $r L$, = $m_{\mathrm{p}} v /(e B)$ (where $v$ is the ion velocity perpendicular to the magnetic field, $m_{\mathrm{p}}$ is the mass of a proton, $e$ is unit charge and $B$ is the magnitude of the magnetic field), could in each case exceed the radius of Mars (due to the high ion velocities concerned). Another difference between the motions of the high energy protons and those of the main population $\mathrm{H}_{\mathrm{sw}}{ }^{+}$protons is that the high energy protons move more closely along the direction of the IMF than along $\boldsymbol{U}_{\mathrm{sw}}$. A third difference between the nominal and high energy protons is that the Lorentz force $\left(e \boldsymbol{v}_{\mathrm{i}} \times \boldsymbol{B}\right)$ could constitute the dominant term (as compared with the $e \boldsymbol{E}$ term) due to the pertaining high ion velocity $\boldsymbol{v}_{\mathrm{i}}$. Under these circumstances, shadowing of the high energy $\mathrm{H}^{+}$ions by Mars can be referred to as "magnetic shadowing" in contrast to the "sw-flow shadowing" of the $\mathrm{H}_{\mathrm{sw}}{ }^{+}$protons previously described.

Figure 5 illustrates how the magnetic shadow depends on proton energy. At relatively low energies, $50 \mathrm{keV}$ (Fig. 5(a)) and $200 \mathrm{keV}$ (Fig. 5(b)), the Martian magnetosphere can scatter the incident ion beams so that part of the scattered trajectories are transposed upstream of the bow shock (left and middle columns). At $600 \mathrm{keV}$ and $3.2 \mathrm{MeV}$ the trajectories of the incident protons are more coherent and these ions start rather to move toward the $-z$ direction due to their gyro-motions. Comparisons between the ion trajectories, especially when they are viewed from the magnetotail (right columns), reveals how the effect of magnetic shadowing differs over the analyzed energy range $50 \mathrm{keV}-3.2 \mathrm{MeV}$. It is noted (not shown) that the ion trajectories of $30 \mathrm{MeV}$ protons were found not to differ substantially from those of 3.2 MeV particles.

Views made from the tail (Fig. 5, right panels) show that the magnetic shadow has a $\pm z$ asymmetry. The energetic ions move toward the $-z$ direction due to the $\left(e \boldsymbol{v}_{\mathrm{i}} \times \boldsymbol{B}\right)$ term in the Lorenz force which points in the $-z$ direction. The $\left(e \boldsymbol{v}_{\mathrm{i}} \times \boldsymbol{B}\right)$ force points toward $-z$ because (a) the magnetic field drapes around Mars and the draped magnetosheath magnetic field has a large component perpendicular to the direction of the undisturbed IMF (see for example figure 7(b) in Kallio et al., 2010) and (b) because the $x$ component of the draped IMF is positive (i.e. opposite to that of the original IMF $x$-component). If the IMF had been oppositely directed, the high energy ions would have gyrated toward the $+z$ direction.

One way to express the hemispherical asymmetry characterizing magnetic shadowing is to use the direction of the undisturbed $\boldsymbol{E}_{\mathrm{sw}}$ which points toward $+z$ in the IMF orientation just described. The asymmetry can then be referred to as the $\pm E_{\mathrm{sw}}$ asymmetry (where high energy ions move toward the $-E_{\mathrm{sw}}$ direction). It is worth noting in this connection that magnetic asymmetry is not caused by the convective electric field but rather by the $\left(e \boldsymbol{v}_{\mathrm{i}} \times \boldsymbol{B}\right)$ force, because the $e \boldsymbol{E}$ force is small as compared with the $\left(e \boldsymbol{v}_{\mathrm{i}} \times \boldsymbol{B}\right)$ force for the high velocity ions analyzed.

It is necessary to study the ion bulk parameters in order to derive a global image of magnetic shadowing. Figure 6 shows the flow lines of the four high energy $\mathrm{H}^{+}$ions and the normalized density $\tilde{n}$ (that is, the density of high energy, say $\mathrm{H}_{\mathrm{h} 1}{ }^{+}$ions, $n\left(\mathrm{H}_{\mathrm{h} 1}{ }^{+}\right)$, divided by their density in the undisturbed solar wind). The number of high energy ions per cell is so low that this density is not very smooth but one can nevertheless draw the following conclusions:

- In the case of $50 \mathrm{keV}$ particles the flow of $\mathrm{H}^{+}$ions is clearly disturbed by the induced magnetosphere of Mars. Similar disturbances can also be seen in the $200 \mathrm{keV}$ case. Density enhancements can be found on the dawn side $(y<0)$ where the ions start to gyrate in the $-z$ direction (see Figs. 6(a) and 6(b), right columns). 



$-25$
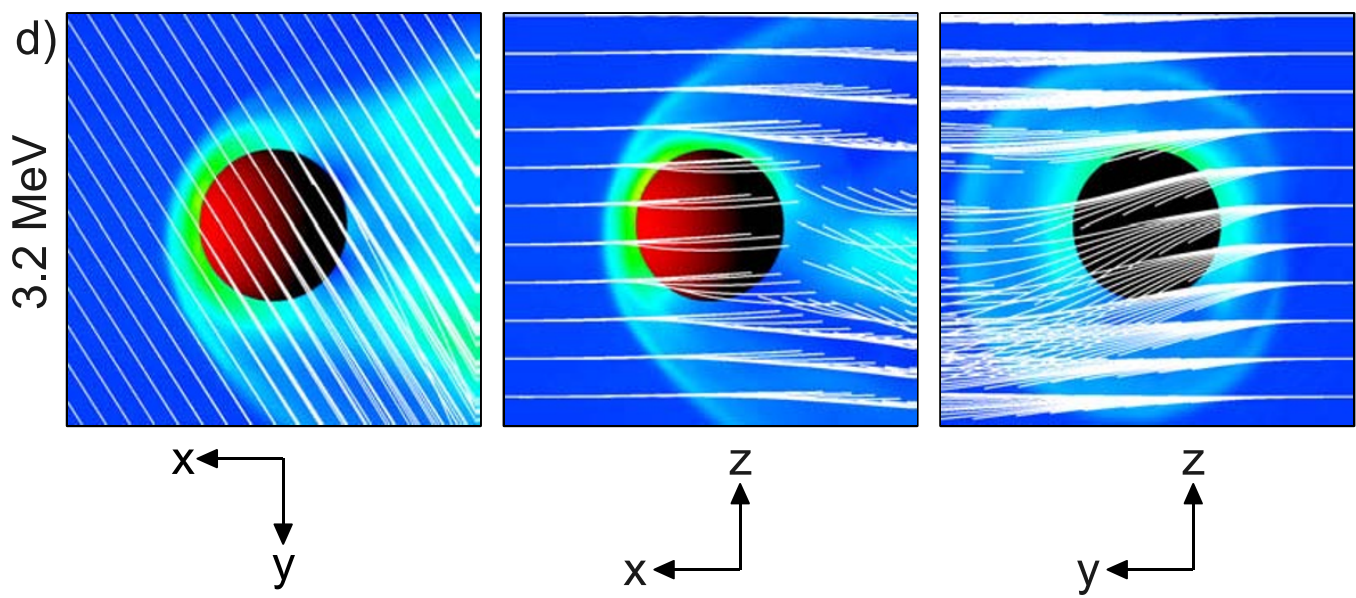

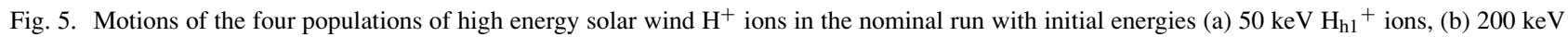
$\mathrm{H}_{\mathrm{h} 2}{ }^{+}$ions, (c) $600 \mathrm{keV} \mathrm{H}_{\mathrm{h} 3}{ }^{+}$ions and (d) $3.2 \mathrm{MeV} \mathrm{H}_{\mathrm{h} 4}{ }^{+}$ions. These ions were generated near the $x=10,000 \mathrm{~km}$ and $y=-18,000 \mathrm{~km}$ planes. The velocity vector of the ions was in the $-\boldsymbol{B}_{\mathrm{sw}}$ direction and their temperature was assumed to be zero. In the panels shown on the left, middle and on the right the view is from above the North Pole; on the dusk side and on the tail side, respectively. The colours provided in the $z=0$ plane (left columns), in the $y=0$ plane (middle columns) and in the $x=0$ plane (right columns) represent the total magnetic field. The white lines show the orbits of high energy $\mathrm{H}^{+}$test particles. The horizontal and the vertical lengths are each from $-10,000 \mathrm{~km}$ to $10,000 \mathrm{~km}$.

- At $600 \mathrm{keV}$ and especially at $3.2 \mathrm{MeV}$ the stream lines are not so greatly displaced from their original directions and, consequently, the low density region (i.e. the overall size of the magnetic shadow) is smaller.
Figure 7 presents the relative densities of various high energy ions near the orbit of Phobos-2 at $r=2.8 R_{\mathrm{M}}$. In this plot the predicted energy dependence of the magnetic shadow is evident in that the size of the magnetic shadow decreases with increasing ion energy. The position of the 


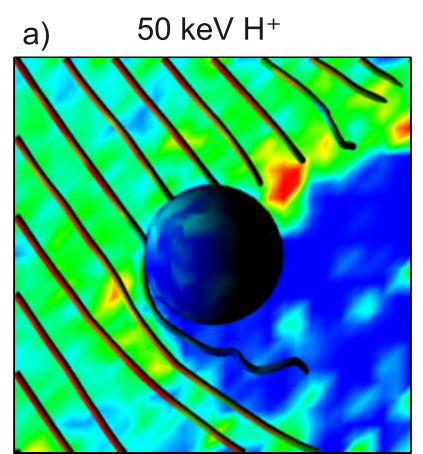

b) $200 \mathrm{keV} \mathrm{H}^{+}$
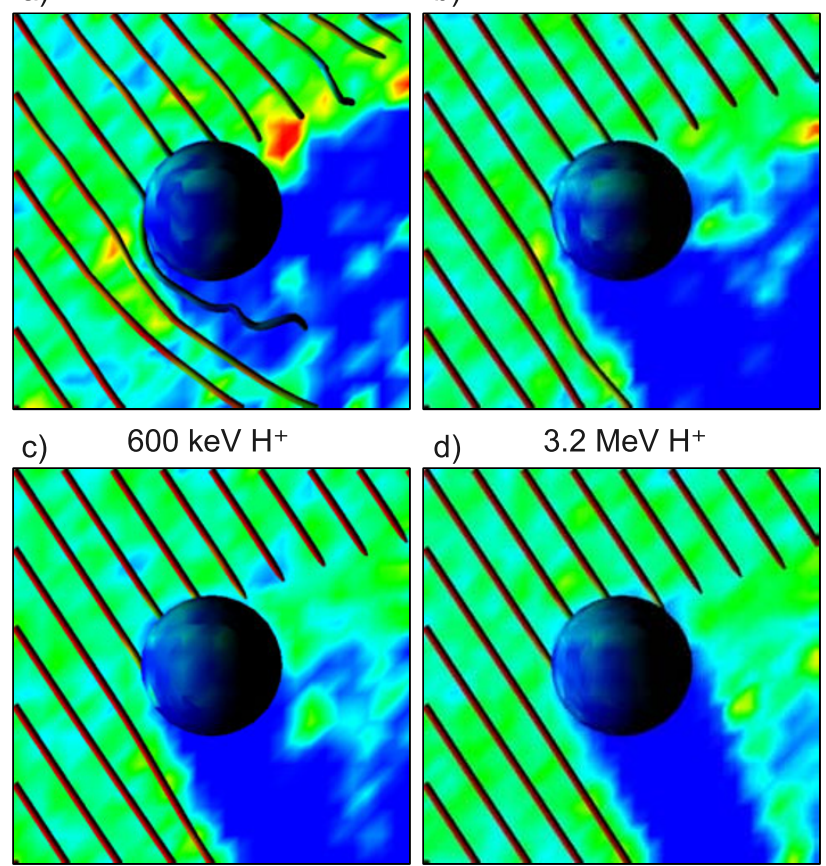

d) $\quad 3.2 \mathrm{MeV} \mathrm{H}^{+}$

normalized

density 0

$\max / 2$
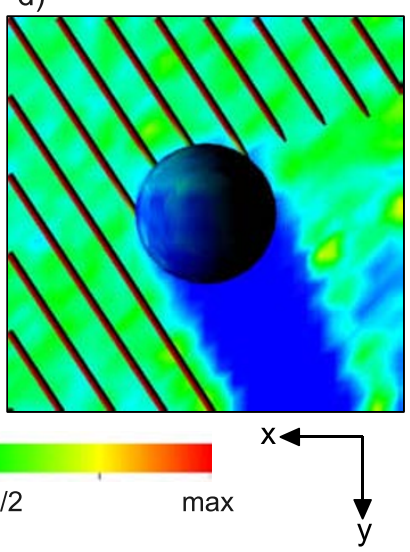

Fig. 6. The flow lines of (a) $50 \mathrm{keV} \mathrm{H} \mathrm{h}_{1}{ }^{+}$ions, (b) $200 \mathrm{keV} \mathrm{H} \mathrm{h}^{+}$ions, (c) $600 \mathrm{keV} \mathrm{H}_{\mathrm{h} 3}{ }^{+}$ions and (d) $3.2 \mathrm{MeV} \mathrm{H}_{\mathrm{h} 4}{ }^{+}$ions near Mars in the nominal run. The colour in the $z=0$ plane and on the $r \sim R_{\mathrm{M}}$ sphere indicates the normalized density of high energy ions, $\tilde{n}$. The horizontal and the vertical lengths are from $-10,000 \mathrm{~km}$ to $10,000 \mathrm{~km}$. Note that some of the stream lines extend below the colour plane to the $z<0$ hemisphere. The enhanced density (red colour) seen in Fig. 6(a) is because the $\boldsymbol{v} \times \boldsymbol{B}$-force impels ions toward the $z<0$ hemisphere (see the text).

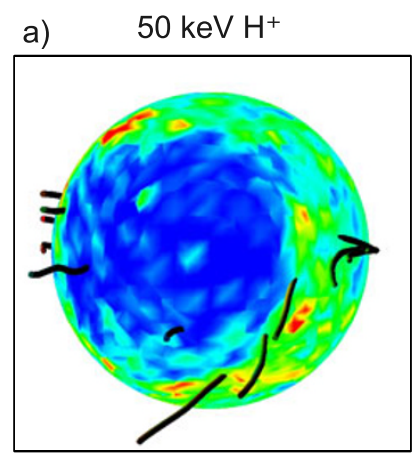

b) $\quad 200 \mathrm{keV} \mathrm{H}^{+}$

c)

$600 \mathrm{keV} \mathrm{H}^{+}$
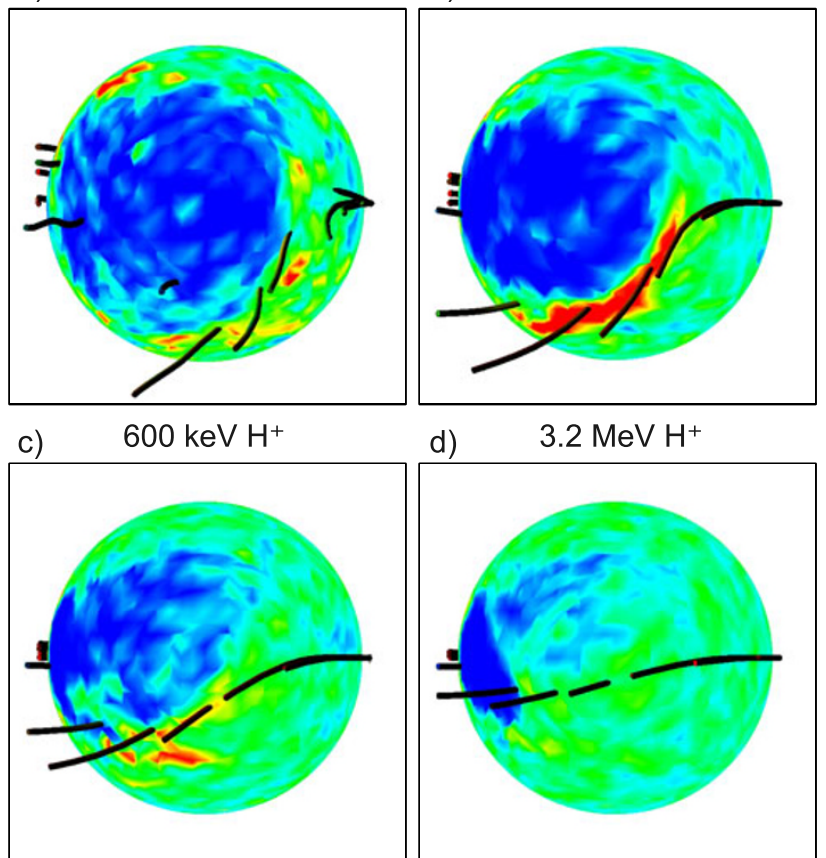

d) $\quad 3.2 \mathrm{MeV} \mathrm{H}^{+}$

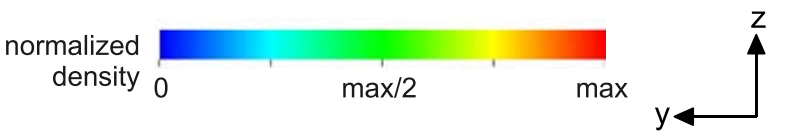

Fig. 7. The normalized density of the high energy $\mathrm{H}^{+}$ions on a spherical shell with radius $r=2.8 R_{\mathrm{M}}$ from the center of Mars (which is approximately the distance at which the Phobos-2 spacecraft measurements were made while following circular orbits). The flow lines are as shown in Fig. 6 and the run is a nominal one. The energies of the $\mathrm{H}^{+}$ions are (a) $50 \mathrm{keV}$, (b) $200 \mathrm{keV}$, (c) $600 \mathrm{keV}$ and (d) $3.2 \mathrm{MeV}$. The view is from the tail toward the Sun. Note how the size of the magnetic shadow (the dark blue region) decreases with increasing energy. 
magnetic shadow displays another dependency on energy in that the magnetic shadow associated with the lowest energy (Fig. 7(b)) is, among the four energies investigated, the one most closely aligned with the solar wind-flow shadow. An increase in energy moves this shadow further along the IMF, that is, the shadow moves toward the dusk side $(y>0)$. It should in addition be noted that the $\pm E_{\mathrm{sw}}$ asymmetry can be identified in Figs. 7(b) and 7(c). This asymmetry, which is associated with the change in direction of the flow lines toward the $z<0$ hemisphere, was previously identified (Fig. 5) in the test particle simulations.

\section{Discussion}

The purpose of this novel paper is to investigate, using HYB modelling, basic characteristics of the Martian high energy proton environment measured by the SLED instrument aboard Phobos-2 (March, 1989).

\subsection{Analysis of the SLED data}

Analysis of the SLED observations is complicated by the fact that this instrument measured protons with energies $<1 \mathrm{MeV}$, the trajectories of which are strongly deviated within the Martian magnetosphere (see Figs. 5(a)5(c)). Moreover, SLED detected ions that were so far away from Mars $\left(r=2.8 R_{\mathrm{M}}\right)$ that their trajectories differed significantly from those followed by particles recorded close to the planet. Further, the SLED telescopes were directed along the nominal Parker IMF direction-a situation which requires the adoption of a plasma model characterized by a non-zero IMF $x$-component (as utilized in the present study).

It is pointed out that, although the analysis presented here provides new insight with regard to the qualitative properties of the high energy particle environment investigated there exists a requirement for more detailed studies. This is because, in the first place the four high energy $\mathrm{H}^{+}$particle populations used in the hybrid model were each assumed to move exactly along the $-\boldsymbol{B}$ direction. In reality, the trajectory of a proton can never be exactly along the IMF because a representative ion moving along $\boldsymbol{B}$ 'feels' the convective electric field $\left(-\boldsymbol{U}_{\mathrm{e}} \times \boldsymbol{B}\right)$ which initially accelerated that ion perpendicular to the magnetic field.

The higher is the energy of an ion, the more closely its velocity can be along $\pm \boldsymbol{B}$. In consequence, the ions detected by SLED in its lowest energy channel $(\sim 30 \mathrm{KeV})$ are characterized by a velocity distribution function that differs significantly from that pertaining in the case of the background ions ( $>30 \mathrm{MeV}$ ) recorded in Channel 6. The role of 'non-beam' velocity distribution functions accordingly now requires to be investigated in detail.

Again, in a stationary situation, the relative energization of different high energy ions ( $>1 \mathrm{MeV}$ ) in the Martian magnetosphere is minor because of the small absolute dimensions of the Mars solar-wind interaction region. For example, if an ion with energy $>1 \mathrm{MeV}$ moves, say, $7000 \mathrm{~km}$ $\left(\sim 2 R_{\mathrm{M}}\right)$ along an $\boldsymbol{E}_{\mathrm{sw}}$ of $1 \mathrm{mV} / \mathrm{m}(=1 \mathrm{~V} / \mathrm{km})$, it will experience a $7 \mathrm{keV}$ 'push' along the electric field, which is small when compared with the initial energy of the ion. Such an energy change was, however, identified during the present modelling when the velocity of $\sim 0.5 \mathrm{MeV}$ protons was derived along the Phobos-2 orbit (figure not shown).
As indicated in Section 5, the size and the shape of the magnetic shadow depends on the strength and direction of the IMF, as well as on the pertaining upstream solar wind plasma parameters. This could be seen when the properties of the high energy proton populations in the four analyzed runs were inter-compared (these figures are not presented here). In one-to-one SLED data/hybrid model comparisons to be made in future, it will be necessary to use realistic upstream parameters in the hybrid model runs. Fortunately, such solar wind plasma parameters are already available in the contemporaneous data of the ASPERA and TAUS instruments flown aboard Phobos-2 (Lundin et al., 1989; Rosenbauer et al., 1989) and complementary magnetic field measurements made by the onboard MAG instrument are also available (Riedler et al., 1989). In future one-to-one comparisons between the in situ measurements and the simulations, the upstream parameters, especially the IMF, should remain unaltered so that the simulated electromagnetic field environment can correspond to the real conditions.

Finally, the attitude of the SLED telescopes must be accurately known in order to correctly interpret any observed decrease/increase in the measured particle fluxes. This is because, while the background Martian electromagnetic environment may not substantially change, the trajectories of particles having energies of several hundred $\mathrm{keV}$ can be substantially deviated by the $\boldsymbol{v} \times \boldsymbol{B}$ force (see Figs. 5 and 7) and this needs to be taken into account.

\subsection{Analysis of the MARIE data}

It is recalled that energetic particle data recorded by the MARIE instrument were earlier studied by Luhmann et al. (2007) using test particle simulations. Here, the electromagnetic field was derived from a 3-D MHD simulation for a case where the $x$-component of the IMF was assumed to be zero. The test particle simulations suggested that the trajectories of $>1 \mathrm{MeV}$ ions follow approximately a straight line in the Martian magnetosheath (which is in agreement with the results of the present study, see Fig. 6(d)).

\section{Conclusions}

The magnetic shadow recorded at Mars by SLED/Phobos-2 during disturbed interplanetary conditions (on 13 March 1989) was simulated using a 3-D hybrid model.

The model predicted that, under certain extreme upstream conditions, a magnetic shadow can be formed that is (a) displaced to the nightside relative to the optical shadow; (b) decreases in size as the particles increase in energy in the $\mathrm{keV}$ to $\mathrm{MeV}$ range; (c) shows an asymmetry in the shadow along the direction of the convective electric field, $\boldsymbol{E}_{\mathrm{sw}}$.

The model also predicted the occurrence of a more well known shadow in the plasma flow on the nightside of Mars (named in this paper the solar wind-flow shadow) and this prediction is confirmed by observations made aboard Phobos- 2 by the ASPERA plasma instrument.

Although the properties of the high energy ions in the model runs displayed a qualitatively similar trend, the quantitative results varied from one run to another. Thus, in order to fully understand the SLED data, it transpires to be necessary to know the actual upstream parameters that were 
present during the taking of the energetic particle measurements.

To determine in detail how upstream conditions affected the energetic particles measured at Mars by SLED, a follow-up study is presently planned in which we will progress from using the hybrid model with test runs as presented here, to inputting to the model ASPERA (plasma density and solar wind velocity data) and MAG (magnetic field) measurements made contemporaneously with the particle records aboard Phobos-2 so as to more closely match the simulated results with the SLED measurements.

Acknowledgments. Figures 3-7 were made using the VisIt open source visualization tool.

\section{References}

Anderson, K. A., R. P. Lin, D. W. Potter, and H. D. Heetderks, An experiment to measure interplanetary and solar electrons, IEEE Trans. Geosci. Electron. GE, 16, 153-156, 1978.

Bertucci, C., C. Mazelle, M. H. Acuňa, C. T. Russell, and J. A. Slavin, Structure of the magnetic pileup boundary at Mars and Venus, J. Geophys. Res., 110, A01209, doi:10.1029/2004JA010592, 2005.

Brain, D., S. Barabash, A. Boesswetter, S. Bougher, S. Brecht, G. Chanteur, D. Crider, E. Dubinin, X. Fang, M. Fraenz, J. Halekas, E. Harnett, M. Holmstrom, E. Kallio, H. Lammer, S. Ledvina, M. Liemohn, K. Liu, J. Luhmann, Y. Ma, R. Modolo, U. Motschmann, H. Nilsson, H. Shinagawa, and N. Terada, A comparison of global models for the solar wind interaction with Mars, Icarus, 206(1), 139-151, doi:10.1016/j.icarus.2009.06.030, 2010.

Cane, H. V. and I. G. Richardson, Interplanetary coronal mass ejections in the near-Earth solar wind during 1996-2002, J. Geophys. Res., 108, 1156, doi:10.1029/2002JA000917, 2003.

Gosling, J. T., Coronal mass ejections and magnetic flux ropes in interplanetary space, in Physics of Magnetic Flux Ropes, edited by Priest, E. R., L. C. Lee, and C. T. Russell, AGU Geophysics Monogram, 58, 343-364, 1990.

Kallio, E., H. Koskinen, S. Barabash, R. Lundin, O. Norberg, and J. G. Luhmann, Proton flow in the Martian magnetosheath, J. Geophys. Res., 99, 23,547-23,559, 1994.

Kallio, E., K. Liu, R. Jarvinen, V. Pohjola, and P. Janhunen, Oxygen ion escape at Mars in a hybrid model: High energy and low energy ions, Icarus, 206, 152-163, doi:10.1016/j.icarus.2009.05.015, 2010.

Luhmann, J. G., C. Zeitlin, R. Turner, D. A. Brain, G. Delory, J. G. Lyon, and W. Boynton, Solar energetic particles in near-Mars space, J. Geophys. Res., 112, E10001, doi:10.1029/2006JE002886, 2007.

Lundin, R., A. Zakharov, R. Pellinen, H. Borg, B. Hultqvist, N. Pissarenko, E. M. Dubinin, S. W. Barabash, I. Liede, and H. Koskinen, First measurements of the ionospheric plasma escape from Mars, Nature, 341, 609-612, doi:10.1038/341609a0, 1989.

Lundin, R., S. Barabash, H. Andersson, M. Holmström, A. Grigoriev, M. Yamauchi, J.-A. Sauvaud, A. Fedorov, E. Budnik, J.-J. Thocaven, D. Winningham, R. Frahm, J. Scherrer, J. Sharber, K. Asamura, H. Hayakawa, A. Coates, D. R. Linder, C. Curtis, K. C. Hsieh, B. R. Sandel, M. Grande, M. Carter, D. H. Reading, H. Koskinen, E. Kallio, P. Riihela, W. Schmidt, T. Säles, J. Kozyra, N. Krupp, J. Woch, J. Luhmann, S. McKenna-Lawler, R. Cerulli-Irelli, S. Orsini, M. Maggi, A. Mura, A. Milillo, E. Roelof, D. Williams, S. Livi, P. Brandt, P. Wurz, and P. Bochsler, Solar wind-induced atmospheric erosion on Mars: First results from ASPERA-3 on Mars Express, Science, 305, 1,933-1,936, 2004.

McKenna-Lawlor, S., V. V. Afonin, K. I. Gringauz, E. Keppler, E. Kirsch, A. Richter, M. Witte, D. O’Sullivan, A. Thompson, A. J. Somogyi, L.
Szabo, and A. Varga, The low energy particle detector SLED $(\sim 30 \mathrm{keV}-$ $3.2 \mathrm{MeV}$ ) and its performance on the Phobos Mission to Mars and its Moons, Nucl. Instr. Meth. Phys. Res. A, 290(1), 217-222, 1990.

McKenna-Lawlor, S. M. P., V. V. Afonin, K. I. Gringauz, E. Keppler, E. Kirsch, A. K. Richter, M. Witte, D. O'Sullivan, A. Thompson, K. Kecskemety, A. Somogyi, L. Szabo, A. Varga, and R. Marsden, Interplanetary variability in interplanetary fluxes recorded by the low energy charged particle detector SLED ( $\sim 30 \mathrm{keV}->30 \mathrm{MeV})$ during the Cruise Phase of the Phobos Mission to Mars and its Moons, Ann. Geophys., 9, 348-356, 1991.

McKenna-Lawlor, S., V. V. Afonin, K. I. Gringauz, K. Kecskemety, E. Keppler, E. Kirsch, A. Richter, P. Rusznyak, K. Schwingenschuh, D. O'Sullivan, A. J. Somogyi, L. Szabo, A. Thompson, A. Varga, Ye Yeroshenko, and M. Witte, Energetic particle studies at Mars by SLED on Phobos 2, Adv. Space Res., 12(9), 231-241, 1992.

McKenna-Lawlor, S. M. P., M. Dryer, C. D. Fry, W. Sun, D. Lario, C. S Deehr, B. Sanahuja, V. Afonin, M. I. Verigin, and G. A. Kotova, Predictions of energetic particle radiation in the close Martian environment, $J$. Geophys. Res., 110, A03102, doi:10.1029/2004JA010587, 2005.

Nagy, A. F., D. Winterhalter, K. Sauer, T. E. Cravens, S. Brecht, C. Mazelle, D. Crider, E. Kallio, A. Zakharov, E. Dubinin, M. Verigin, G. Kotova, W. I. Axford, C. Bertucci, and J. G. Trotignon, The plasma environment of Mars, Space Sci. Rev., 111(1), 33-114, 2004.

Reames, D. V., Particle acceleration at the Sun and in the heliosphere, Space Sci. Rev., 90, 413-491, 1999.

Riedler, W., D. Möhlmann, V. N. Oraevsky, K. Schwingenschuh, Ye Yeroshenko, J. Rustenbach, Oe. Aydogar, G. Berghofer, H. Lichtenegger, M. Delva, G. Schelch, K. Pirsch, G. Fremuth, M. Steller, H. Arnold, T. Raditsch, U. Auster, K.-H. Fornacon, H. J. Schenk, H. Michaelis, U. Motschmann, T. Roatsch, K. Sauer, R. Schröter, J. Kurths, D. Lenners, J. Linthe, V. Kobzev, V. Styashkin, J. Achache, J. Slavin, J. G. Luhmann, and C. T. Russell, Magnetic fields near Mars: first results, Nature, 341, 604-607, 1989.

Rosenbauer, H., N. Shutte, A. Galeev, K. Gringauz, and I. Apathy, Ions of Martian origin and plasma sheet in the Martian magnetosphere: initial results of the TAUS experiment, Nature, 341, 612-614, doi:10.1038/341612a0, 1989.

Sagdeev, R. Z. and A. V. Zakharov, Brief history of the Phobos Mission, Nature, 341, 581-585, 1989.

Vignes, D., C. Mazelle, H. Reme, M. H. Acuňa, J. E. P. Connerney, 'R. P. Lin, D. L. Mitchell, P. Cloutier, D. H. Crider, and N. F. Ness, The solarwind interaction with Mars: locations and shapes of the Bow Shock and the Magnetic Pile-up Boundary from the observations of the MAG/ER experiment onboard Mars Global Surveyor, Geophys. Res. Lett., 27(1), 49-52, 2000.

Winningham, J. D., R. A. Frahm, J. R. Sharber, A. J. Coates, D. R. Linder, Y. Soobiah, E. Kallio, J. R. Espley, R. Lundin, S. Barabash, M. Holmstrom, H. Andersson, M. Yamauchi, A. Grigoriev, J. R. Scherrer, S. J. Jeffers, D. O. Kataria, J. U. Kozyra, J. G. Luhmann, E. C. Roelof, D. J. Williams, S. Livi, C. C. Curtis, K. C. Hsieh, B. R. Sandel, H. Koskinen, T. Sales, P. Riihela, W. Schmidt, M. Grande, M. Carter, J. A. Sauvaud, A. Fedorov, J. J. Thocaven, S. McKenna-Lawlor, S. Orsini, R. CerulliIrelli, M. Maggi, P. Wurz, P Bochsler, N. Krupp, J. Woch, A. Franz, K. Asamura, and C. Dierker, Electron oscillations in the induced Martian magnetosphere, Icarus, 182(2), 360-370, 2006.

Zeitlin, C., T. Cleghorn, F. Cucinotta, P. Saganti, V. Anderson, K. Lee, L. Pinsky, W. Atwell, R. Turner, and G. Badhwar, Overview of the Martian radiation environment experiment, Adv. Space Res., 33(12), 2,204-2,210, 2004.

S. McKenna-Lawlor (e-mail: stil@nuim.ie), E. Kallio, R. Jarvinen, and V. V. Afonin 